EPJ Web of Conferences 60, 20056 (2013)

DOI: $10.1051 /$ epjconf $/ 20136020056$

(C) Owned by the authors, published by EDP Sciences, 2013

\title{
Tuning of PYTHIA6 to Minimum Bias Data
}

\author{
Nameeqa Firdous ${ }^{1, a}$, Gerald Rudolph ${ }^{1}$ \\ ${ }^{1}$ Institute of Astro and Particle Physics, University of Innsbruck, Austria
}

\begin{abstract}
New Minimum Bias tunes of Multiple Parton Interaction parameters of PYTHIA6 are presented to the Minimum Bias published data from ATLAS and CDF II using three different PDFs. These tunes describe the selected data, especially pt distribution of ATLAS data, reasonably well for all three selected PDFs including MC adapted PDF set.
\end{abstract}

\section{Introduction}

Most of the hadronic interactions at high energies are inelastic and predominantly soft interactions. Current QCD models, for example the PYTHIA6 Monte Carlo event generator [1], attempt to simulate these by allowing several parton-parton scatterings to occur within a single hadron-hadron collision. In this study six parameters are found to be sensitive to the selected data and considered as adjustable parameters and were tuned to the minimum bias data published by ATLAS Collaboration [3] at 0.9 and $7 \mathrm{TeV}$ and from $\mathrm{CDF}$ II at $1.96 \mathrm{TeV}$ [10] using three different PDF types: Leading order (CTEQ5L) [8], MC adapted (MRSTMCAL or LO**) [9], Next-to-leading order (CT10) [5] . The results are compared to the latest ATLAS tune AMBT2B [4] for PYTHIA6.

\section{Multiple Parton Interaction Parameters}

The main parameter of the multiple parton interactions (MPI) model is the infrared regularization parameter pt0(PARP $(82))$. Its energy evolution is controlled by PARP(90). Within the new interleaved MPI/ISR scenario we have chosen option $\operatorname{MSTP}(82)=5$ for the matter overlap in the varying impact parameter model which is controlled by a single parameter, $d=\operatorname{PARP}(83)$. Colour annealing scenario has been selected $(\operatorname{MSTP}(95)=6)$ in which the amount of colour reconnection in the final state is controlled by parameter PARP(78). One more parameter PARP(77) which provides suppression of CR for fast moving strings is also found to be sensitive to the data. But we fixed this parameter at 0.90 in the final step of tuning to eliminate instability found in fit results. In addition two QCD parameters, $\Lambda_{M E}(\operatorname{PARP}(1))$ and $\Lambda_{F S R}$, are also treated as adjustable parameters in contrast to [7].

\section{Tuning Method}

The method employed to tune the parameters in this study can be found in [2]. It is based on a linear and iterative approach and allows the simultaneous variation of many parameters. It has been developed and extensively used within ALEPH and has been adapted for pp or ppbar reactions [7]. The parton shower and hadronisation parameters have been determined independently from fits to e+e- $\rightarrow$ hadrons data collected at $Z^{0}$ pole by the ALEPH experiment [2].

\section{Results}

Tuned values of six parameters considered for simultaneous variation in this paper are listed in Table 2 for three different PDF sets. Distributions used in this study are from diffraction limited phase spaces and are listed in Table 1. Figure 1 shows the comparison plots of three new tunes and AMBT2B using MC adapted PDF set to the ATLAS multiplicity and eta distributions. It is shown that all the three types of PDF sets including MC adapted PDF set $\mathrm{LO}^{* *}$, describe $\mathrm{Nch}$ equally well in contrast to the AMBT2B tunes. All tunes describes eta distribution quite well and AMBT2B shows perfect agreement for high multiplicity as compared to new tunes but this discrepancy is not very significant. Figure 2 shows comparison plots for $\mathrm{pt}$ and average pt vs Nch distribution. It can be seen that pt distribution is quite well described by all three new tunes in contrast to the AMBT2B. This is achieved by treating $\Lambda_{M E}$ and $\Lambda_{F S R}$ as adjustable parameters in addition to the four other MPI parameters. The pt spectrum for $p t>3 \mathrm{GeV}$ is now well described also in case of MC adapted PDF (LO**), which is not the case for ATLAS tune AMBT2B [4]. Average pt vs Nch is a very sensitive distribution, all three new tunes describe this quantity perfectly whereas AMBT2B is lower than data which means this tune predicts more activity than required by data, this effect can also be seen in the multiplicity distribution. Figure 3 shows comparison plots for Nch and pt distribution

\footnotetext{
ae-mail: nameeqa@yahoo.com
} 


\begin{tabular}{ll}
\hline Observable & Weights \\
\hline Track based MB Data at 7TeV & \\
\hline$N_{c h}$, track $p_{\perp}>500 \mathrm{MeV}, N_{c h} \geq 6$ & 40 \\
$p_{\perp}$, track $p_{\perp}>500 \mathrm{MeV}, N_{c h} \geq 6$ & 40 \\
$\eta$, track $p_{\perp}>500 \mathrm{MeV}, N_{c h} \geq 6$ & 40 \\
$\left\langle p_{\perp}\right\rangle$ vs. $N_{c h}, p_{\perp}>500 \mathrm{MeV}, N_{c h} \geq 1$ & 40 \\
\hline$N_{c h}$, track $p_{\perp}>2500 \mathrm{MeV}, N_{c h} \geq 1$ & 20 \\
$p_{\perp}$, track $p_{\perp}>2500 \mathrm{MeV}, N_{c h} \geq 1$ & 20 \\
$\eta$, track $p_{\perp}>2500 \mathrm{MeV}, N_{c h} \geq 1$ & 20 \\
\hline$N_{c h}$, track $p_{\perp}>100 \mathrm{MeV}, N_{c h} \geq 20$ & 10 \\
$p_{\perp}$, track $p_{\perp}>100 \mathrm{MeV}, N_{c h} \geq 20$ & 10 \\
$\eta$, track $p_{\perp}>100 \mathrm{MeV}, N_{c h} \geq 20$ & 10 \\
\hline Track based MB Data at $\mathbf{0 . 9 T e V}$ & \\
\hline$N_{c h}$, track $p_{\perp}>500 \mathrm{MeV}, N_{c h} \geq 6$ & 20 \\
$p_{\perp}$, track $p_{\perp}>500 \mathrm{MeV}, N_{c h} \geq 6$ & 20 \\
$\eta$, track $p_{\perp}>500 \mathrm{MeV}, N_{c h} \geq 6$ & 20 \\
$\left\langle p_{\perp}\right\rangle$ vs. $N_{c h}, p_{\perp}>500 \mathrm{MeV}, N_{c h} \geq 1$ & 20 \\
\hline$N_{c h}$, track $p_{\perp}>2500 \mathrm{MeV}, N_{c h} \geq 1$ & 10 \\
$p_{\perp}$, track $p_{\perp}>2500 \mathrm{MeV}, N_{c h} \geq 1$ & 10 \\
$\eta$, track $p_{\perp}>2500 \mathrm{MeV}, N_{c h} \geq 1$ & 10 \\
\hline$N_{c h}$, track $p_{\perp}>100 \mathrm{MeV}, N_{c h} \geq 20$ & 5 \\
$p_{\perp}$, track $p_{\perp}>100 \mathrm{MeV}, N_{c h} \geq 20$ & 5 \\
$\eta$, track $p_{\perp}>100 \mathrm{MeV}, N_{c h} \geq 20$ & 5 \\
\hline CDF Run II minimum bias & \\
\hline$\left\langle p_{\perp}\right\rangle v s . N_{c h}$, track $p_{\perp}>400 \mathrm{MeV}$ & 20 \\
\hline
\end{tabular}

Table 1. Minimum Bias observables with weights used in the tunes to minimum bias data from ATLAS and CDF II.

\begin{tabular}{lccc}
\hline Parameter & CTEQ5L & MRSTMCAL & CT10 \\
\hline PARP(82) & 2.92 & 2.67 & 2.09 \\
PARP(90) & 0.25 & 0.22 & 0.18 \\
PARP(83) & 1.75 & 1.72 & 1.70 \\
PARP(78) & 0.42 & 0.43 & 0.51 \\
PARP(1) & 0.17 & 0.07 & 0.18 \\
PARP(72) & 0.52 & 0.50 & 0.46 \\
\hline
\end{tabular}

Table 2. Tuning result of MB fit for three PDF sets

for other phase space. pt description is very good for new tunes and AMBT2B again unable to describe this distribution. All tunes describe multiplicity distribution for this phase space in a same way but description is rather bad. Table 2 shows that MC adapted PDF requires abnormally small value of $\Lambda_{M E} \sim 70 \mathrm{MeV}$ to describe the selected data. This parameter is very sensitive to the selected PDF types but is strongly correlated to the pt 0 .

\section{Conclusion}

New tunes of PYTHIA6 to minimum bias published data at three center of mass energies, ATLAS at 0.9, 7TeV and CDFII at $1.96 \mathrm{TeV}$ are presented including first minimum bias tune using NLO PDF. Equally good descriptions of minimum bias distributions, especially the pt distributions, are obtained for all three types of PDFs, which was found impossible in the ATLAS tune AMBT2B [4].

All plots are produced using Rivet toolkit [6].

\section{References}

[1] Torbjörn Sjöstrand, Stephen Mrenna, and Peter Z. Skands. PYTHIA 6.4 Physics and Manual. JHEP, 05:026, 2006.

[2] R. Barate et al. Studies of quantum chromodynamics with the ALEPH detector. Phys.Rept., 294:1-165, 1998.

[3] G. Aad et al. Charged-particle multiplicities in pp interactions measured with the ATLAS detector at the LHC. New J.Phys., 13:053033, 2011.

[4] Atlas tunes of pythia 6 and pythia 8 for mc11. Technical Report ATL-PHYS-PUB-2011-009, CERN, Geneva, Jul 2011.

[5] Hung-Liang Lai, Marco Guzzi, Joey Huston, Zhao Li, Pavel M. Nadolsky, et al. New parton distributions for collider physics. Phys.Rev., D82:074024, 2010.

[6] Andy Buckley, Jonathan Butterworth, Leif Lonnblad, Hendrik Hoeth, James Monk, et al. Rivet user manual. 2010.

[7] Nameeqa Firdous and Gerald Rudolph. PYTHIA MPI model tuning to hadron collider data: Preliminary results. Nucl.Phys.Proc.Suppl., 207-208:73-76, 2010.

[8] H.L. Lai et al. Global QCD analysis of parton structure of the nucleon: CTEQ5 parton distributions. Eur.Phys.J., C12:375-392, 2000.

[9] A. Sherstnev and R.S. Thorne. Parton Distributions for LO Generators. Eur.Phys.J., C55:553-575, 2008.

[10] T. Aaltonen et al. Measurement of Particle Production and Inclusive Differential Cross Sections in $\mathrm{p}$ anti-p Collisions at $\mathrm{s}^{* *}(1 / 2)=1.96-\mathrm{TeV}$. Phys.Rev., D79:112005, 2009. 

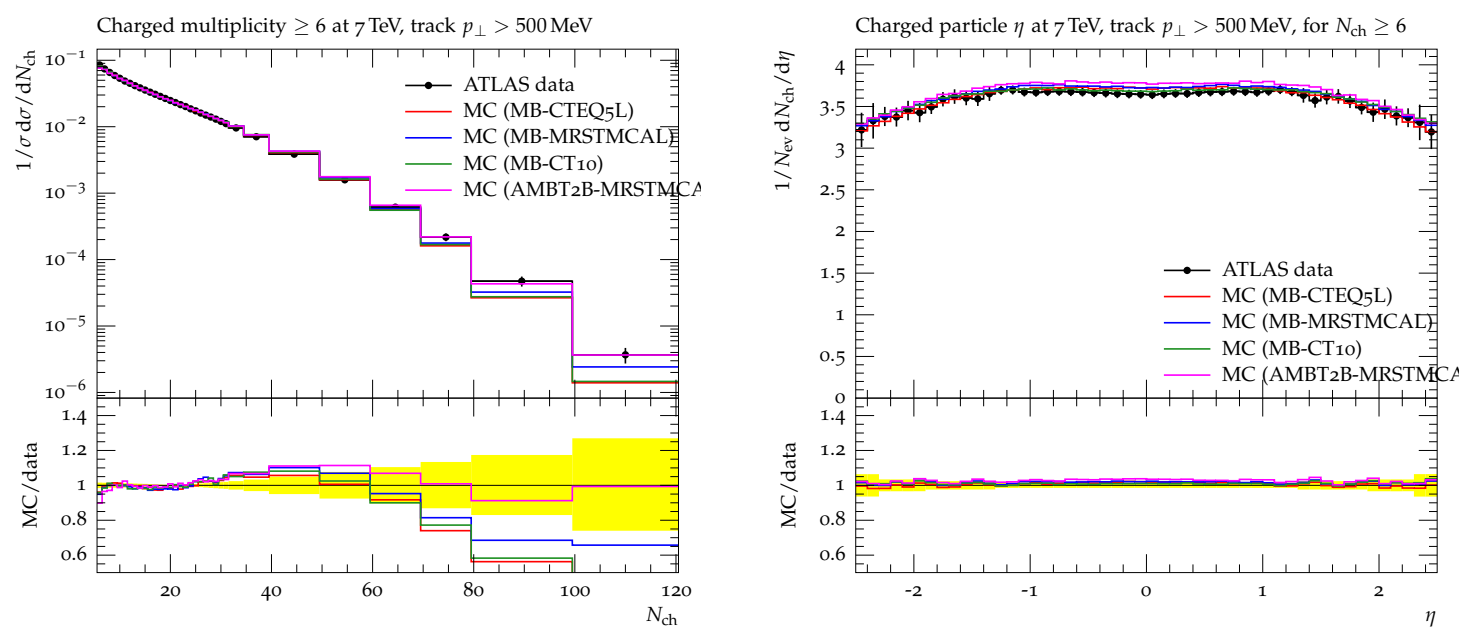

Figure 1. Comparison plots of new tunes and AMBT2B to ATLAS minimum bias data, at 7TeV: Multiplicity and eta distribution
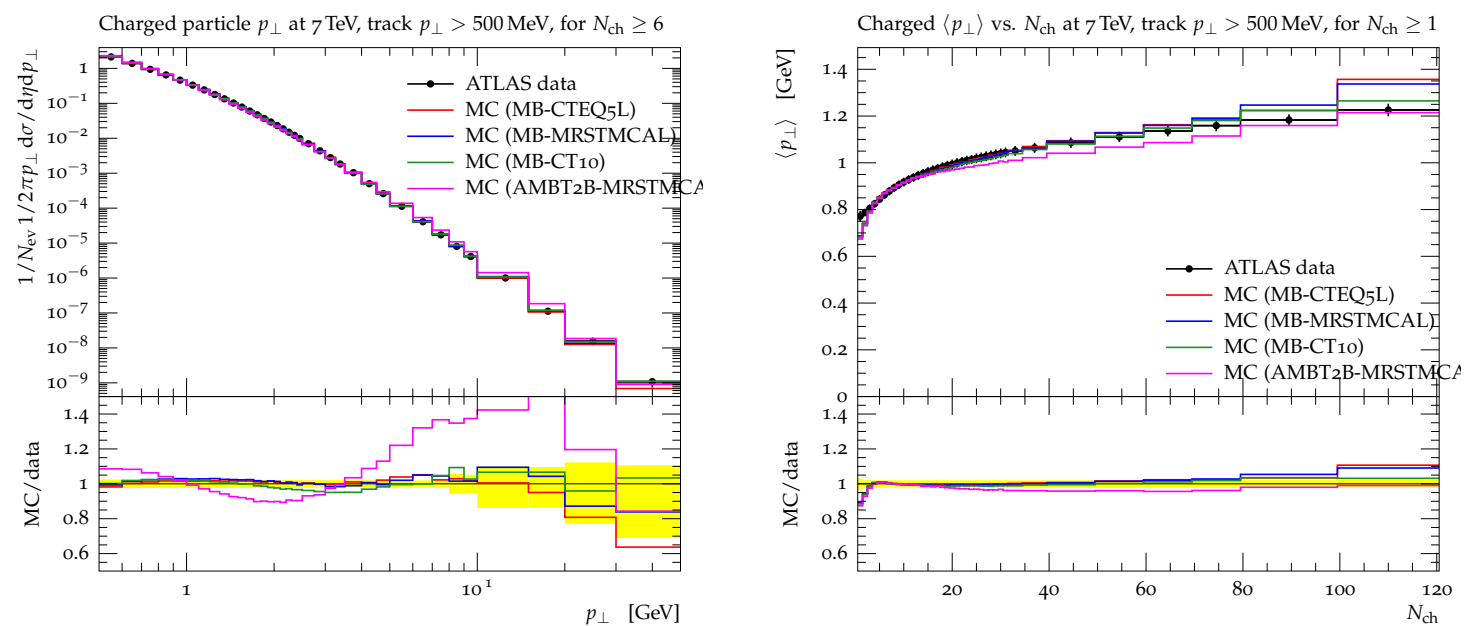

Figure 2. Comparison plots of new tunes and AMBT2B to ATLAS minimum bias data at 7TeV: Transverse momentum pt and average pt vs Nch distribution
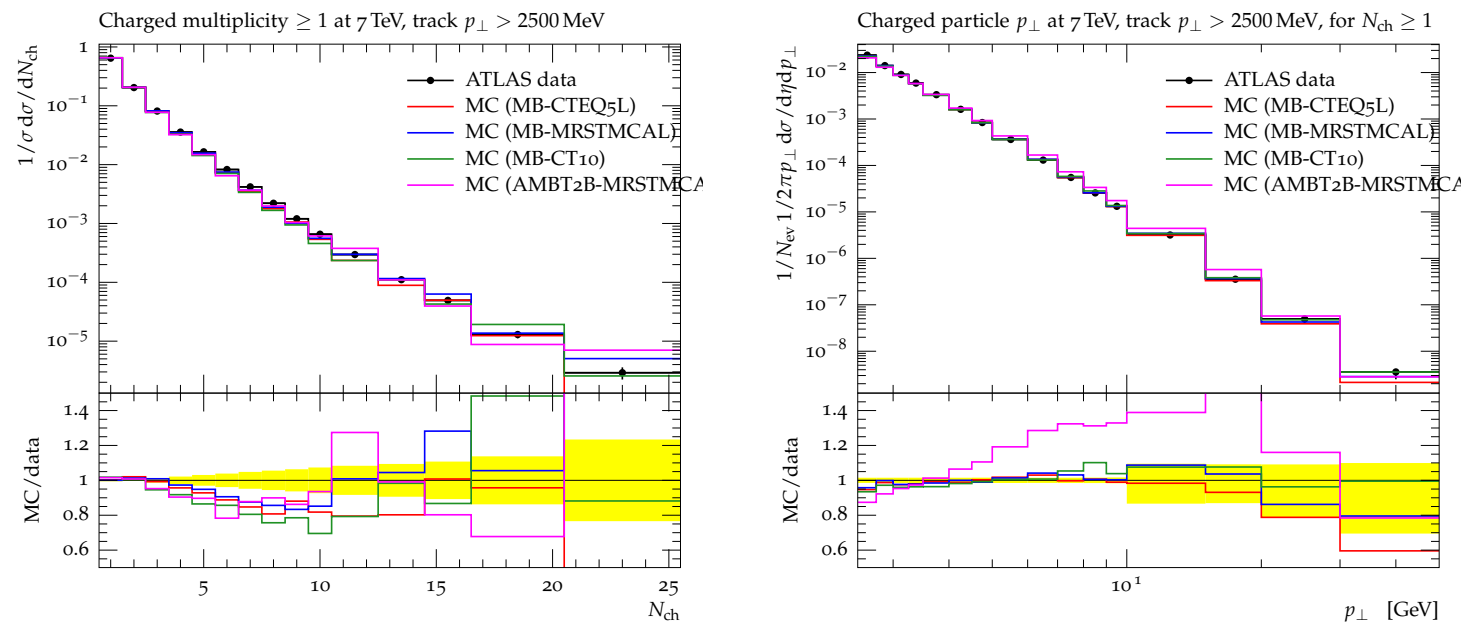

Figure 3. Comparison plots of new tunes and AMBT2B to ATLAS minimum bias data at 7TeV: Multiplicity and pt distribution 